\title{
Efeito da seleção no primeiro ciclo de postura para produção de ovos sobre o desempenho no segundo ciclo
}

\author{
Improvement in second cycle egg production traits as a response of first cycle selection in leghorn layers
}

\author{
Gilberto Silber Schmidtt ${ }^{1,2}$ Elsio Antonio Pereira de Figueiredo ${ }^{1,2}$
}

\section{RESUMO}

Utilizou-se uma população selecionada $(C C) e$ uma controle (CCc), para avaliar o efeito da seleção, no primeiro ciclo de postura, durante 5 gerações, para características produtivas, sobre o desempenho no segundo ciclo. Avaliou-se o peso corporal (PC) na $16^{\underline{a}}$ (PC16), 40 (PC40), 75a $(P C 75)$ e 111a (PC111) semana, peso médio do ovo (PMO), idade à maturidade sexual (IMS), produção de ovos ave/dia (POAD) da IMS até a 64ํㅗ semana, a idade de reinício da postura após a muda (RP), a POAD do RP até a $120^{a}$ semana, a relação altura x largura dos ovos (REL) e a densidade (DENS). Exceto PC111 e REL, as demais características avaliadas no segundo ciclo apresentaram mudanças genéticas significativas decorrentes da seleção para as mesmas características no primeiro ciclo. Houve incremento na POAD e PC e redução na IMS no primeiro ciclo. No segundo ciclo, foram mantidas as diferenças entre CC e CCc, para as características POAD e IMS, indicando resposta correlacionada entre estas características no primeiro e segundo ciclo. Conclui-se que a seleção para aumento do desempenho produtivo das aves durante o primeiro ciclo de postura mostrou ser eficiente para elevar os níveis de produção no segundo ciclo.

Palavras-chave: produção de ovos, qualidade do ovo, muda forçada, melhoramento, resposta correlacionada.

\section{ABSTRACT}

An experiment was carried out to evaluate the effect of first cycle selection on second cycle egg production traits. The traits evaluated were body weight at 16 (PC16), 40 (PC40), 75 (PC75) and 111 (PC111) weeks of age; average egg weight (PMO); age at on set production (IMS); hen day egg production (POAD) from IMS up to 64 weeks of age; age at first egg after moulting (RP); POAD from RP up to 120 weeks of age; egg shape index (REL) and density (DENS). Except PC111 and REL all traits were significantly influenced by first cycle selection. The genetic changes increased in egg production and body weight and decreased in age at onset egg prodution. Differences in egg production and age at onset production between $C C$ and $C C$ c remained in second cycle, indicating a correlated response between these traits in first and second cycles. It was concluded that selection in first cycle also improved egg production in second laying cycle.

Key words: egg production, egg quality, forced moulting, breeding, correlated response.

\section{INTRODUÇÃO}

A utilização do segundo ciclo de postura, após muda forçada de penas, em poedeiras comerciais, é prática utilizada amplamente pelos produtores, quando economicamente viável. Embora ocorra redução no custo de reposição do plantel, o desempenho no segundo ciclo de postura, em termos de produção e eficiência alimentar, e qualidade do ovo são inferiores ao do primeiro ciclo. A produção de ovos no segundo ciclo normalmente é $20 \%$ inferior ao obtido. A inclusão nos índices de seleção dos recordes obtidos, no segundo ciclo, poderia reduzir esta diferença (FAIRFULL, 1982).

Entre os critérios de seleção utilizados inclui-se apenas o desempenho das aves durante o primeiro ciclo de produção, impedindo a utilização de genes que atuam tardiamente (FAIRFULL \& GOWE, 1990). Alguns trabalhos, visam determinar os efeitos

${ }^{1}$ Zootecnistas, pesquisadores da Embrapa Suínos e Aves, CP. 21, 89700-000, Concórdia, SC, Brasil. E-mail: schmidt@cnpsa.embrapa.br. Autor para correspondência.

${ }^{2}$ Bolsistas do $\mathrm{CNPq}$ 
genéticos da idade na performance das aves, tanto para o primeiro como para o segundo ciclo, vem sendo conduzidos por LILJEDHAL et al (1994). Porém, a literatura apresenta pouca informação sobre a influência da seleção por desempenho no primeiro ciclo e a resposta correlacionada no segundo ciclo.

GOWE \& FAIRFULL (1982) verificaram que os efeitos genéticos importantes no primeiro também o são no segundo ciclo. Em seus estudos, com exceção da gravidade especifica e do peso corporal, as linhagens selecionadas apresentaram, no segundo ciclo, resultados superiores à população controle, indicando resposta correlacionada entre os ciclos. A produção de ovos, peso do ovo, gravidade específica, peso corporal e eficiência alimentar apresentaram, respectivamente, correlação entre o primeiro e segundo ciclo, de 0,$71 ; 0,86 ; 0,85 ; 0,85$ e 0,76 .

Objetivou-se com o presente trabalho avaliar a influência da seleção, baseada na performance das aves no primeiro ciclo, sobre o desempenho no segundo ciclo.

\section{MATERIAL E MÉTODOS}

O experimento foi conduzido no Centro Nacional de Pesquisa de Suínos e Aves (CNPSA) da EMBRAPA, localizado em Concórdia, SC. Utilizaram-se observações referentes a uma linhagem de postura White Leghorn (CC), selecionada durante 5 gerações (1992/1996) para aumento da produção e do peso de ovo, redução da idade à maturidade sexual, redução do peso corporal e uniformização da relação altura x largura do ovo, no primeiro ciclo de postura, e uma população controle (CCc), originada de CC em 1984.

Foram utilizadas 1762 e 510 femêas, respectivamente, das linhas $\mathrm{CC}$ e CCc do plantel de seleção, que foram criadas em piso até a 16ª semana de idade, quando foram transferidas para gaiolas individuais. $\mathrm{O}$ manejo nas fases de cria, recria e produção foi o recomendado para a linhagem EMBRAPA-011. As rações continham $2.850 \mathrm{kcal}$ EM.kg-1 e 20\% PB, 2.750kcal EM.kg-1 e $14 \%$ PB, 2.850kcal EM.kg ${ }^{-1}$ e $16 \%$ PB e $2.800 \mathrm{kcal} \mathrm{EM}^{\mathrm{k}} \mathrm{kg}^{-1}$ e $15 \%$ $\mathrm{PB}$, respectivamente, para as fases inicial (0-6 semanas), crescimento (7-18 semanas), produção I (1945 semanas) e produção II (acima de 46 semanas).

Na 64ํㅗ semana de idade, após a expansão das populações, um total de 1.648 e 484 fêmeas, respectivamente, das linhas $\mathrm{CC}$ e $\mathrm{CCc}$, foram submetidas à muda forçada, segundo metodologia descrita por AVILA (1994). No primeiro ciclo de postura, avaliou-se o peso corporal na 16 (PC16) e 40a (PC40) semanas, o peso médio do ovo (PMO), mensurado na $24^{\mathrm{a}}, 28^{\mathrm{a}}, 32^{\mathrm{a}}, 36^{\mathrm{a}}, 42^{\mathrm{a}}, 48^{\mathrm{a}}, 56^{\mathrm{a}}$ e $64^{\mathrm{a}}$ semana, a idade à maturidade sexual (IMS) e a produção de ovos ave/dia (POAD) da IMS até a 64르 semana de idade. No segundo ciclo, foram avaliados o peso corporal na 75을 (PC75) e 111 (PC111) semanas, PMO, mensurados na $81^{a}$, $93^{a}, 106^{a}$ e $111^{\underline{a}}$ semana de idade, a idade de reinício da postura após a muda (RP), a POAD a partir de RP até a 120 a semana, a relação altura $\mathrm{X}$ largura dos ovos (REL) e a densidade dos ovos (DENS).

Considerando que $\mathrm{CC}$ e $\mathrm{CC}$ tiveram a mesma base genética, a diferença entre ambas foi considerada como mudança genética. Para determinação das correlações entre as características de interesse, utilizou-se apenas a linhagem CC. Os dados foram analisados utilizando-se o pacote estatístico SAS.

\section{RESULTADOS E DISCUSSÃO}

As análises das variâncias e as médias estimadas para as características produção de ovos ave/dia (POAD), peso médio do ovo (PMO), peso corporal na 16 (PC111) semana de idade, a idade a maturidade sexual (IMS), retorno à postura após muda (RP), relação altura $\mathrm{x}$ largura dos ovos (REL) e densidade (DENS) são apresentados nas tabelas 1 e 2 .

A produção de ovos, no primeiro ciclo, foi 18,83 (CC) e $24,56 \%$ (CCc) superior ao segundo ciclo (Figura 1), confirmando os resultados obtidos por AVILA(1994). Em ambos os ciclos, a produção de ovos foi superior para a linhagem selecionada, indicando ganhos genéticos no processo de seleção e, também, redução na magnitude das diferenças entre os ciclos $(23,33 \%)$. As diferenças entre CC e CCc de 10,31 e $15,64 \%$, respectivamente, para o primeiro e segundo ciclo, indicam resposta correlacionada da seleção por desempenho no primeiro (POAD1) sobre o desempenho no segundo ciclo (POAD2). A correlação estimada entre POAD1 e POAD2 foi de 0,34 (Tabela 3), resultado inferior ao valor de 0,71 obtido por GOWE \& FAIRFULL(1982).

Com relação à IMS e RP verifica-se o mesmo efeito, com ganhos de 3,05 dias para IMS e 1,91 dias para RP. A correlação entre IMS e RP foi próxima de zero (Tabela 3), indicando que o retorno após a muda depende do método de muda utilizado e não da idade à maturidade sexual, confirmando os resultados obtidos por AVILA (1994).

À exceção de PC111, o peso corporal foi mais elevado na linha selecionada, em $15 \mathrm{~g}$ na $16^{\mathrm{a}}, 61 \mathrm{~g}$ 
Tabela 1 - Quadrados médios e coeficiente de variação obtidas para peso médio do ovo (PMO), produção de ovos ave/dia (POAD), peso corporal (PC), idade a maturidade sexual (IMS), retorno a postura após a muda (RP), relação altura x largura do ovo (REL) e densidade (DENS).

\begin{tabular}{|c|c|c|c|c|c|c|}
\hline \multirow{2}{*}{ Caract. } & \multicolumn{3}{|c|}{ Primeiro ciclo } & \multicolumn{3}{|c|}{ Segundo ciclo } \\
\hline & LINHA & RESÍDUO & CV (\%) & LINHA & RESÍDUO & CV $(\%)$ \\
\hline PMO & $11,05^{*}$ & 46,00 & 5,80 & $391,62^{*}$ & 19,59 & 6,97 \\
\hline POAD & $22015,19^{*}$ & 77,27 & 11,25 & & & \\
\hline $\mathrm{PC}^{1}$ & $89426,81^{*}$ & 8620,85 & 8,58 & $515171,26^{*}$ & 36694,75 & 13,65 \\
\hline $\mathrm{PC}^{2}$ & $1437348,65^{*}$ & 29724,35 & 10,29 & $1720,70^{\mathrm{NS}}$ & 68199,18 & 12,90 \\
\hline IMS/RP (d) & $3518,34^{*}$ & 100,93 & 6,72 & $1356,74^{*}$ & 58,77 & 18,69 \\
\hline REL & - & - & - & $36,38^{\mathrm{ns}}$ & 24,63 & 4,78 \\
\hline DENS & - & - & - & $15,33^{*}$ & 2,15 & 51,58 \\
\hline
\end{tabular}

Para o primeiro ciclo na $16^{\mathrm{a}}$ e $40^{\mathrm{a}}$ semana (1) e para o segundo ciclo na $75^{\mathrm{a}}$ e $111^{\mathrm{a}}$ semana. (2)

na $40^{\mathrm{a}}$ e $37 \mathrm{~g}$ na $75^{\mathrm{a}}$ semanas de idade, do que na controle. Considerando que a correlação entre peso corporal e produção de ovos é negativa (LEDUR et al., 1993), o aumento no peso das aves, reduziu, em parte, o ganho genético para produção de ovos. O PC16 apresentou correlação ao redor de 0,30 com os pesos nas demais idades, resultado este inferior ao valor de 0,85 obtido por GOWE \& FAIRFULL (1982). Já o PC40 apresentou correlações de 0,58 e 0,70 , respectivamente, com PC75 e PC111 e, PC75 de 0,70 com PC111 (Tabela 3). Portanto, o peso da ave, antes da muda, apresenta correlação maior com os pesos no segundo ciclo de produção.

O PMO no primeiro ciclo foi 6,07g (CC) e $6,75 \mathrm{~g}(\mathrm{CCc})$ inferior ao segundo ciclo (Figura 2). Em relação à população controle, $\mathrm{CC}$ reduziu o $\mathrm{PMO}$, que foi, em média, 0,34 e 1,02g menor que CC, respectivamente, no primeiro e segundo ciclo, principalmente devido à maior ênfase dada à seleção para POAD. A correlação entre o peso do ovo entre os ciclos $(0,70)$ está próxima da estimativa obtida por GOWE \& FAIRFULL (1982), o que favorece uma resposta correlacionada, conforme verificado no presente trabalho, da seleção no primeiro sobre o segundo ciclo.

A REL não apresentou ganho genético significativo em relação à população controle. A correlação desta característica só foi significativa com o peso médio do ovo no primeiro $(0,27)$ e segundo ciclo $(0,46)$. Já para DENS, verificaram-se ganhos genéticos na ordem de 0,21 e correlações negativas, significativas, com a produção de ovos no primeiro $(-0,13)$ e segundo ciclos $(-0,24)$.

O peso corporal, nas diversas idades, apresentou correlação entre 0,22 e 0,31 com o peso médio do ovo em ambos os ciclos. A correlação entre

Tabela 2 - Médias obtidas e os respectivos erros-padrão para as características para peso médio do ovo (PMO), produção de ovos ave/dia (POAD), peso corporal (PC), idade a maturidade sexual (IMS), retorno a postura após a muda (RP), relação altura x largura do ovo (REL) e densidade (DENS).

\begin{tabular}{|c|c|c|c|c|}
\hline \multirow{2}{*}{ Características } & \multicolumn{2}{|c|}{ Primeiro ciclo } & \multicolumn{2}{|c|}{ Segundo ciclo } \\
\hline & $\mathrm{CCc}$ & $\mathrm{CC}$ & $\mathrm{CCc}$ & $\mathrm{CC}$ \\
\hline PMO (g) & $57,52 \pm 3,48^{a}$ & $57,18 \pm 3,28^{b}$ & $64,27 \pm 4,63^{\mathrm{A}}$ & $63,25 \pm 4,36^{\mathrm{B}}$ \\
\hline POAD (\%) & $72,32 \pm 10,12^{b}$ & $79,78^{\mathrm{a}} \pm 8,36^{\mathrm{a}}$ & $58,06 \pm 17,7^{\text {В }}$ & $67,14 \pm 14,34^{A}$ \\
\hline $\mathrm{PC}^{1}(\mathrm{~g})$ & $1070 \pm 76^{\mathrm{b}}$ & $1085 \pm 97^{\mathrm{a}}$ & $1431 \pm 207^{\mathrm{A}}$ & $1394 \pm 186^{\text {B }}$ \\
\hline $\mathrm{PC}^{1}(\mathrm{~g})$ & $1628 \pm 182^{b}$ & $1689 \pm 169^{\mathrm{a}}$ & $2025 \pm 278^{\mathrm{A}}$ & $2023 \pm 256^{\mathrm{A}}$ \\
\hline $\mathrm{IMS} / \mathrm{RP}(\mathrm{d})^{2}$ & $151,7 \pm 10,42^{\mathrm{a}}$ & $148,75 \pm 9,93^{\mathrm{b}}$ & $42,47 \pm 8,21^{\mathrm{A}}$ & $40,56 \pm 7,50^{\mathrm{B}}$ \\
\hline REL & - & - & $1,34 \pm 0,06^{\mathrm{A}}$ & $1,34 \pm 5,64^{\mathrm{A}}$ \\
\hline DENS & - & - & $2,68 \pm 1,40^{\mathrm{B}}$ & $2,89 \pm 1,48^{\mathrm{A}}$ \\
\hline
\end{tabular}

${ }^{1}$ Para o primeiro ciclo na $16^{\mathrm{a}}$ e $40^{\mathrm{a}}$ semana e para o segundo ciclo na $75^{\mathrm{a}}$ e $111^{\mathrm{a}}$ semana.

Letras diferentes, dentro de ciclo e característica, diferem estatisticamente $(\mathrm{P}, 0,01)$ 
Tabela 3 - Correlação entre as diversas características avaliadas no primeiro e segundo ciclo de postura.

\begin{tabular}{|c|c|c|c|c|c|c|c|c|c|c|c|}
\hline & IMS & PC16 & PC40 & PC75 & PC111 & $\mathbf{R P}$ & REL & DENS & POAD1 & POAD2 & PMO1 \\
\hline PC16 & $-0,13$ & - & & & & & & & & & \\
\hline PC40 & 0,20 & 0,32 & - & & & & & & & & \\
\hline PC75 & 0,09 & 0,31 & 0,58 & - & & & & & & & \\
\hline PC111 & 0,09 & 0,33 & 0,70 & 0,70 & - & & & & & & \\
\hline $\mathrm{RP}$ & 0,06 & $-0,10$ & $-0,05$ & $-0,42$ & 0,00 & - & & & & & \\
\hline REL & 0,00 & 0,13 & 0,13 & 0,09 & 0,20 & 0,00 & - & & & & \\
\hline DENS & 0,00 & 0,00 & 0,00 & 0,00 & 0,00 & 0,07 & $-0,09$ & - & & & \\
\hline POAD1 & 0,17 & 0,00 & 0,00 & 0,00 & 0,00 & 0,05 & 0,00 & $-0,13$ & - & & \\
\hline POAD2 & 0,07 & 0,06 & 0,06 & 0,00 & $-0,05$ & $-0,11$ & 0,00 & $-0,24$ & 0,34 & - & \\
\hline PMO1 & 0,22 & 0,23 & 0,31 & 0,22 & 0,22 & $-0,05$ & 0,27 & 0,00 & 0,00 & 0,00 & - \\
\hline $\mathrm{PMO} 2$ & 0,14 & 0,23 & 0,27 & 0,22 & 0,28 & 0,00 & 0,46 & 0,00 & 0,00 & $-0,10$ & 0,70 \\
\hline
\end{tabular}

${ }^{1,2}$ Primeiro e segundo ciclo, respectivamente.

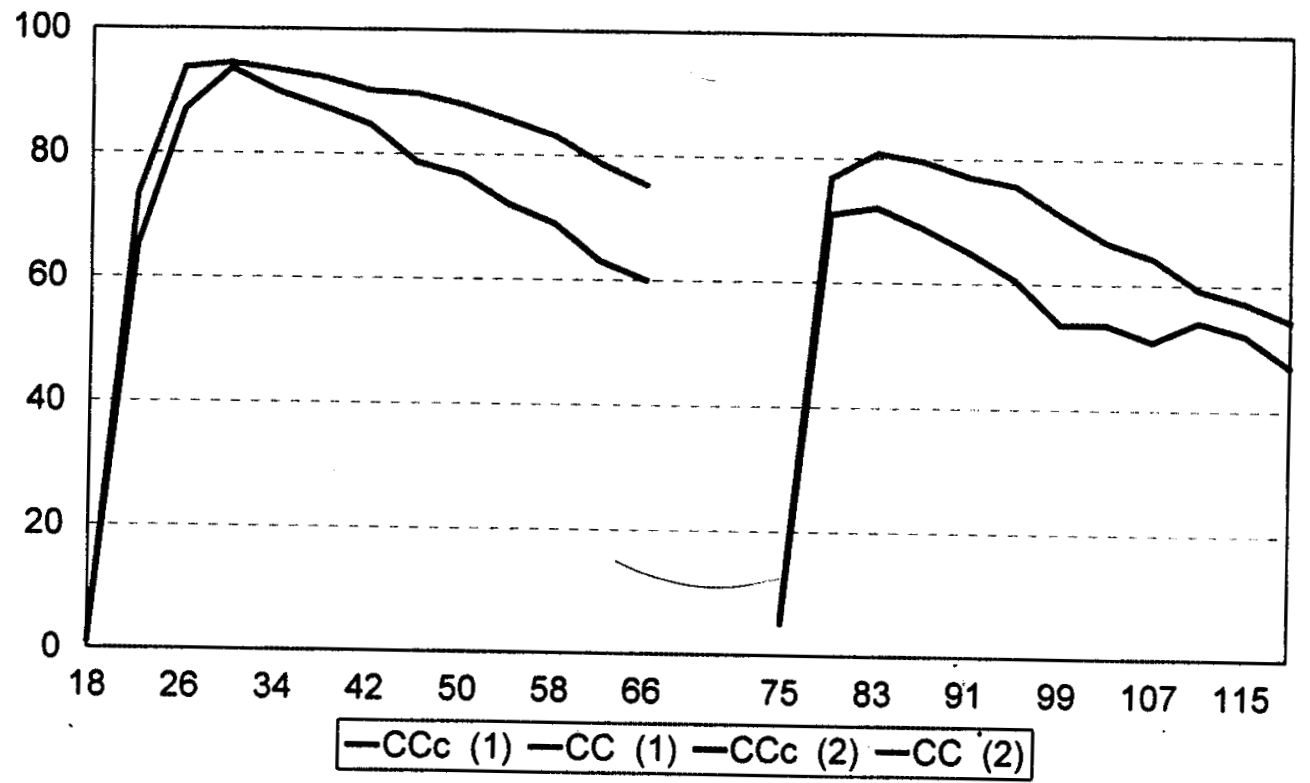

Figura 1 - Produção de ovos ave/dia obtida para o primeiro e segundo ciclo de postura

IMS e as carcterísticas POAD e PMO no primeiro ciclo e PMO no segundo ciclo foi, respectivamente, 0,17 ; 0,22 e 0,14 .

No geral, a exceção das correlações entre os pesos corporais nas diversas idades e o peso médio do ovo no primeiro e segundo ciclo, as demais correlações foram não significativas ou de baixa magnitude.

\section{CONCLUSÃO}

A seleção para melhoria no primeiro ciclo de postura das aves mostrou ser eficiente para elevar os níveis de produção no segundo ciclo. Porém, a ampla utilização de múltiplos ciclos na produção comercial e a baixa correlação $(0,34)$ entre a produção de ovos no primeiro e segundo ciclo, a médio prazo, 


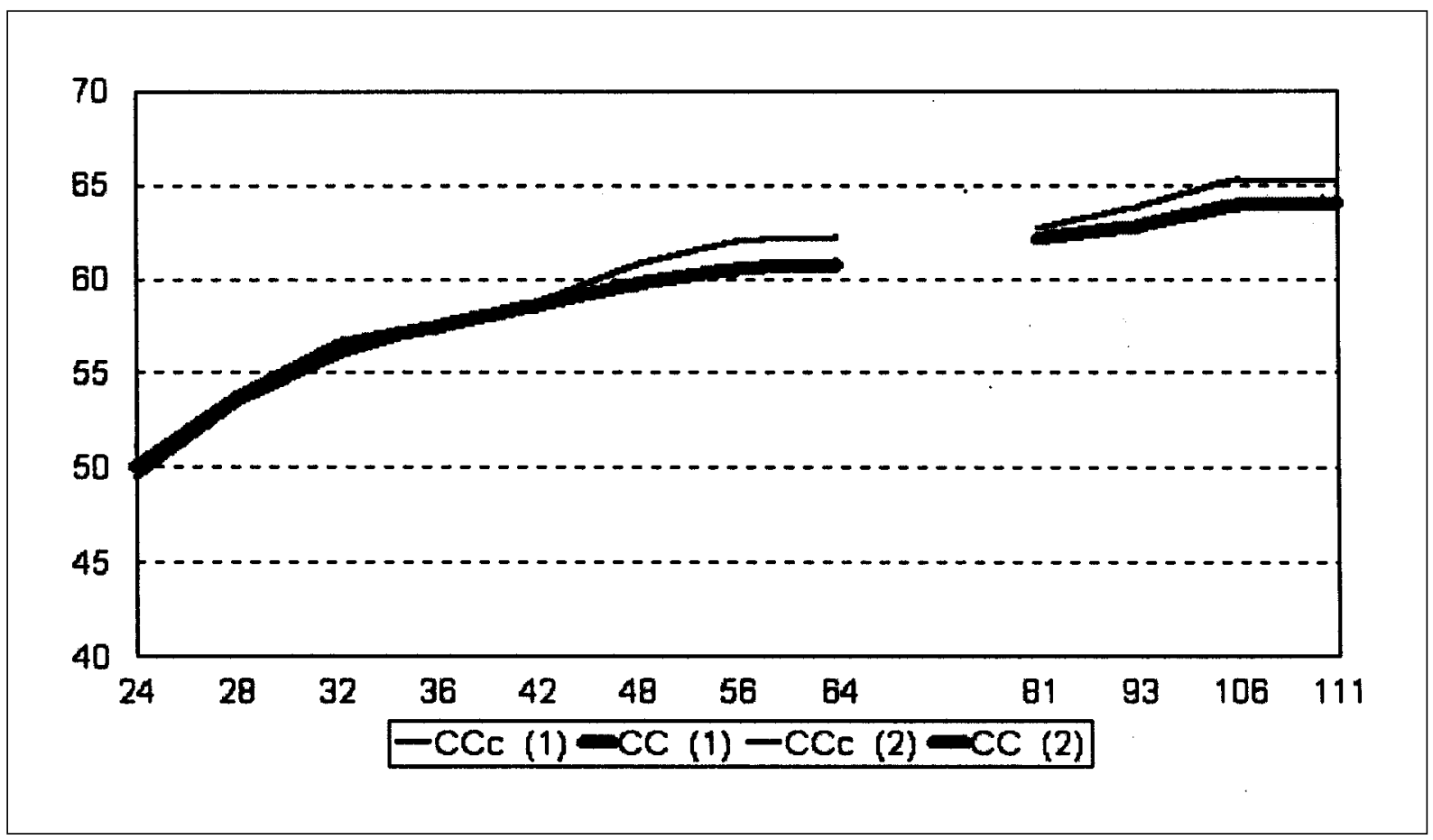

Figura 2 - Peso médio do ovo obtido para o primeiro e segundo ciclo de postura

determinará a necessidade de inclusão do desempenho das aves no segundo ciclo no processo de seleção, reduzindo as diferenças entre os ciclos.

\section{REFERÊNCIAS BIBLIOGRÁFICAS}

AVILA, V.S. Programa de muda forçada para poedeiras comerciais. Concórdia : EMBRAPA-CNPSA, 1994. 2p. (EMBRAPA-CNPSA. Comunicado Técnico, 212).

FAIRFULL, R.W. Combining ability, heterosis and reciprocal effects for first and second year performance in six selected Leghorn strains crossed in a complete diallel. In: ANNUAL NATIONAL BREEDERS' ROUNDTABLE, 31., 1982, St. Louis, Missouri. Proceedings ... St. Louis : ni., 1982. p.119-137.
FAIRFULL, R.W.; GOWE, R.S. Genetics of egg production in chickens. In: CRAWFORD, R.D. (ed.). Poultry breeding and genetics. Amsterdam : Elsevier Science Publishers, 1990. p.705-759.

GOWE, R.S.; FAIRFULL, R.W. Heterosis in egg-type chickens. In: WORLD CONGRESS OF GENETICS APPLIED OF LIVESTOCK PRODUCTION, 2., 1982, Guelph,. Proceedings... Guelph : ni., 1982. p.121-132.

LEDUR, M.C. et al. Parâmetros genéticos e fenotípicos para características produtivas em linhagens de poedeiras de ovos brancos. Pesquisa Agropecuária Brasileira, v.28, n.9, p.1031-1037, 1993.

LILJEDHAL, L.E. et al. Age changes in genetic and enviromental variation in laying hens. Theoretical and Applied Genetetics, v.67, p.391-401, 1994. 